\title{
A CASE STUDY APPROACH TO INTRODUCE CIRCULAR ECONOMY IN SUSTAINABLE DESIGN EDUCATION
}

\author{
Ivan ESPARRAGOZA ${ }^{1}$ and Jaime MESA-COGOLLO ${ }^{2}$ \\ ${ }^{1}$ Penn State University \\ 2Universidad Tecnologica de Bolivar
}

\begin{abstract}
Finding sustainable solutions to worldwide problems has become a critical challenge for engineers. The use of natural resources and the generation of materials now degradable should be controlled when sustainable solutions are pursued. As a result, the notion of reusing materials and products for longer periods is gaining momentum in the design for sustainability, and the circular economy is becoming the new paradigm to be implemented for that purpose. In contrast to the traditional linear economy that is based on making, using and disposing of products, the circular economy is focused on remanufacturing, reusing and recycling products and materials to extend their life. However, the circular economy is not formally introduced in the academia, and future engineers are not getting prepared to incorporate it into the design for sustainability. This work aims to propose a pedagogical approach to introduce the concept of circular economy in engineering design. A case study is presented to compare the design of a product based on the linear economy model (manufacture, use, waste) and the redesign of the product using the circular economy model (remanufacture, reuse, recycle). The comparison will be attained using sustainability performance indicators considered from early design stages. The contrast between the original design based on the linear economy and the re-design using the circular economy allows illustrating the benefits of the circular economy model to enhance the sustainability performance of a product.
\end{abstract}

Keywords: Circular economy, sustainable design, education

\section{INTRODUCTION}

The circular economy concept is a trending topic regarding the sustainability of products and services. Environmental benefits from circular economy such as emissions reduction, recyclability, and reuse of products are remarked as common strategies included in the philosophy of manufacturing companies through reusable designs and the use of biodegradable or recyclable manufacturing materials. However, the benefits of circular economy on sustainability are not widely known yet. This situation can be originated by several factors such as: a) the novelty of the concept, which only have 10 years, b) the need of additional behaviour change from the perspective of user/consumer about sustainability impacts [1] and c) lack of legal pressure and policies promoting circular economy activities such as reuse, recycling, remanufacture and repair [2].

From the perspective of engineering, the circular economy is a prominent research topic. However, it is in many institutions not formally considered into engineering design curricula, which enables the main abilities and knowledge necessary to develop the product design process. Therefore, this article aims to establish a first approach in the learning processes for engineering students about circular economy. The proposed method consists of a module based case study analysis, which demonstrates the relevance of circular products compared to conventional (linear) products. Sustainability and circularity indicators are also established to assess different scenarios from the case study analysis. This approach will help students to better understand and incorporate circularity during the Life Cycle Analysis (LCA) to assess the design of products in upper-level courses, and further applications. 


\section{PROPOSED METHODOLOGY}

\subsection{Linear vs. Circular Economy concepts}

The proposed learning module initiates with a diagnosis about previous knowledge regarding linear economy and circular economy models and their differences. This diagnosis aims to identify if students can understand the type of products developed in each model considering their sustainability performance. The diagnosis will be performed through a survey to identify the degree of domain about the topics of interest. Once the previous knowledge diagnosis is applied, a lecture can be developed highlighting the most confusing concepts and misconceptions founded in the diagnosis. Moreover, it is preferable that linear and circular economy concepts are exemplified using conventional daily products such as disposable and non-recyclable products.

From the perspective of sustainability, it is necessary to explain to students the two main strategies to increase the sustainability performance of products: Closing and Slowing Resource Flows. The first implies the change from an open and linear path to a complete circular loop. Therefore, material flow is returned into a useful lifecycle. On the other hand, Slowing Resource Flows denotes to extend the useful life as much as possible. The understanding of these strategies is critical to promote sustainability into the students learning process, especially respect to the most desirable scenario, which is achieved when both strategies are simultaneously implemented. Figure 1 shows the different scenarios obtained from both Closing and Slowing Resource Flows.

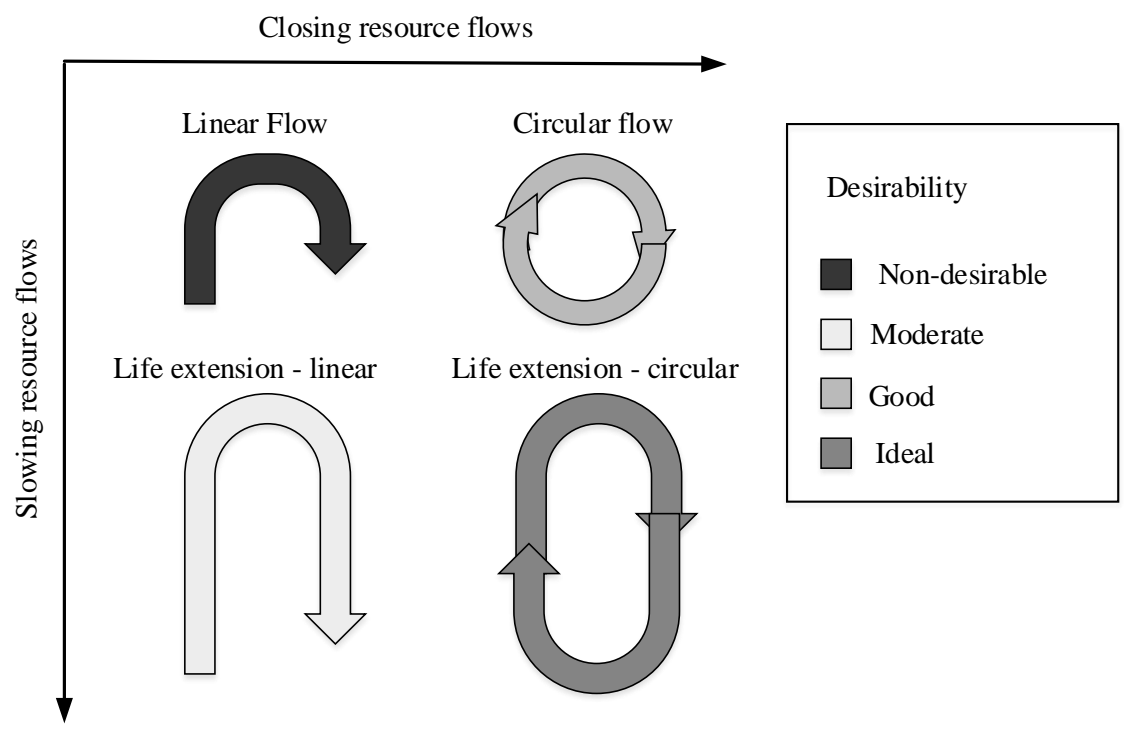

Figure 1. Slowing and Closing resources flow relationship. Adapted from Brocken et al. [3]

The second phase of the proposed method consists of the selection of a case study product, which provides useful information about the degree of understanding and assimilation of circular economy concepts. It is preferable to choose a short-life product to generate important improvements in sustainability and achieve better persuasive results on student thinking. Two preferable conditions are suggested to choose the case study product:

Condition 1: Non-recyclable products or demanding highly complex recycling process

Condition 2: Non-reusable products (e.g., disposable products)

It is important to clarify that any product can involve circularity at a certain degree, where the better demonstration of the proposed method is achieved through a low-circularity case study product. In that way, students are stimulated to consider the concept of circular economy from common daily activities.

\subsection{Circularity and Sustainability diagnosis of the case study product}

Once the case study product is chosen, it is necessary to assess its initial circularity and sustainability performance. The initial diagnosis of circularity is done through sustainability indicators based on the recyclable, reusable and re-manufacturable mass of the case study product. In the same way, it is possible to measure conventional sustainability indicators such as Energy Consumption, Carbon 
Footprint, and Product Cost. Those indicators can be calculated for material production, manufacturing, and final disposal stages. To provide a generic set of circularity and sustainability indicators, Table 1 summarises the indicators suggested in this learning module.

Table 1. List of indicators for circularity and sustainability measurement

\begin{tabular}{cll}
\hline Subject & Dimensions & \multicolumn{1}{c}{ Indicators } \\
\hline \multirow{2}{*}{ Circularity } & & $\begin{array}{l}\text {-Recyclable Mass }(\mathrm{kg}) \\
\text {-Reusable Mass }(\mathrm{kg}) \\
\end{array}$ \\
& & -Biodegradable Mass (kg) \\
\hline \multirow{2}{*}{ Sustainability } & & $\begin{array}{l}\text {-Energy Consumption (MJ) } \\
\end{array}$ \\
& Economical & -Carbon Footprint (J) \\
& -Product Cost (USD) \\
\hline
\end{tabular}

\subsection{Interventions to increase circularity}

After the circularity and sustainability diagnosis, the next step consists of implementing interventions to improve the indicators considered. Such interventions are based on four main necessary attributes of circular products design principles according to the Ellen McArthur Foundation [4].

1. Reusing, refurbishing or remanufacturing products, parts, and materials without incurring in meaningful changes.

2. Keeping products, components, and materials in use as long as possible to increase the useful life and avoid replacement of products in short lifecycles.

3. Diversifying the use of products, components, and materials for additional purposes when they can no longer be used for the original purpose.

4. Using pure, non-toxic or at least easily separated inputs into products to minimise cost for recycling. From the product attributes previously mentioned, four main interventions or improvement alternatives are established based on the manufacturing material of the case study product. The proposed method is limited to apply interventions focused exclusively on the change of manufacturing material and its consequent implications on the circularity and sustainability of the product. The four proposed interventions are described in detail below. It is suggested that each intervention can be considered as a separated alternative to provide several comparisons and discussion scenarios. Error! Reference source not found. 2 summarises the flow scenarios generated for each proposed intervention on the original case study product.

Intervention 1 - Reusable Material: This alternative consists of extending the useful life of the product through a non-recyclable material that allows the reuse of the product.

Intervention 2 - Recyclable Material: In this intervention, the material is converted from waste into a source of raw material for subsequent re-processing, although non-recyclable waste can be generated depending on the recycling process efficiency.

Intervention 3- Biodegradable Material: In this third alternative, the replacing manufacturing material must be biodegradable, which provides closure in the material flow but towards a state prior to raw material.

Intervention 4-Recyclable and Reusable Material: Select a recyclable material that allows the reuse of the product. This alternative seeks to obtain the maximum positive impact on the circularity and sustainability indicators. 
Table 2. Material flow scenarios for original product alternatives

\begin{tabular}{l}
\hline Life extension - linear \\
Case Study Product \\
Intervention 1
\end{tabular}

\subsection{Sustainability comparison between the case study and alternatives}

Once the interventions are implemented on the case study product, the next step is to quantitatively compare the circularity and sustainability indicators. Such comparison can be done graphically, using bar or radial charts. From a holistic perspective, the best alternative will be the one that denotes lower values in the conventional sustainability indicators and higher values in the circularity indicators proposed.

\section{CASE STUDY}

This section describes the implementation of the proposed method though a case study product. The results obtained from this case study are based on engineering data from the CES Selector Software distributed by Granta Design. Therefore, calculation values showed in this work are accurate and can be replicated without restriction.

\subsection{Selection of the case study product}

The case study product consists of a disposal cup made of paper and polyethylene, which commonly is employed a single time. According to Ziada [5], by 2010 in the USA approximately 16 billion of disposable cups were sent to landfill. Although many cups are disposed in recycling cans, it is not possible to recycle them because their design makes the separation between paper and polyethylene difficult and most of them are dirty, causing rejection into the process [6]. Figure 2 shows a typical disposable cup. The mass of a cup including the main body and plastic covering is 12.1 grams, where paper cup represents $12 \mathrm{~g}$ and plastic covering $0.1 \mathrm{~g}$.

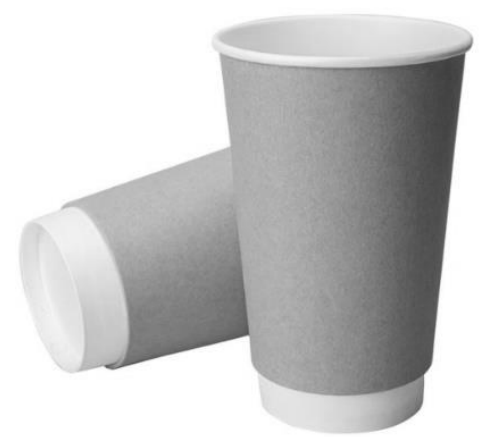

Figure 2. Case study - disposal cup (paper + PE)

\subsection{Circularity and sustainability diagnosis}

Table 3 summarises the values of circularity and sustainability indicators described previously for the chosen case study product. 
Table 3. Values for circularity and sustainability indicators

\begin{tabular}{|l|l|c|}
\hline Dimensions & Indicator & Value \\
\hline \multirow{4}{*}{ Circularity } & Recyclable Mass (kg) & 0 \\
\cline { 2 - 3 } & Reusable Mass (kg) & 0 \\
\cline { 2 - 3 } & Biodegradable Mass (kg) & 0 \\
\hline \multirow{3}{*}{ Sustainability } & Energy Consumption (MJ) & 0.885 \\
\cline { 2 - 3 } & Carbon Footprint (kg) & 0.028 \\
\cline { 2 - 3 } & Product Cost (USD) & 0.25 \\
\hline
\end{tabular}

\subsection{Design interventions to increase circularity}

After the diagnosis in circularity and sustainability diagnosis, the next step is to generate replacing alternatives according to the four interventions previously described in subsection 2.4. In this way, recyclable, biodegradable and reusable manufacturing materials are proposed to replace the manufacturing material from the case study product. Table 4 summarises the four proposed alternatives that can be given by the instructor or can be generated by students under instructor supervision.

Table 4. Proposed interventions - product examples

\begin{tabular}{|c|c|c|c|}
\hline \multicolumn{3}{|c|}{ Proposed Interventions } \\
\hline 1 & 2 & 3 & \\
& & & \\
& & & \\
\hline & & & \\
\hline & PLA & Porthchole & \\
\hline
\end{tabular}

Once the alternative manufacturing materials are proposed, students proceed to measure the same indicators of circularity and sustainability for each alternative. It is important to highlight that the information gathering process about energy consumption and carbon footprint among others can be complex and heterogeneous in terms of data search process on the internet. Table 5 comprises the values of indicators for the case study product and the alternatives 1 to 4 . The Styrofoam and PLA cups are bought in lots of large quantities bringing the unitary cost down. The porcelain and stainless-steel cups are bought in single units. The costs in Table 5 were obtained from commercial sites on the internet.

Table 5. Overall comparison between the original product and proposed alternatives

\begin{tabular}{|c|l|c|c|c|c|c|}
\hline \multirow{2}{*}{ Dimensions } & \multicolumn{1}{|c|}{ Indicator } & Original & \multicolumn{4}{c|}{ Alternatives } \\
\cline { 4 - 7 } & & Product & $\mathbf{1}$ & $\mathbf{2}$ & $\mathbf{3}$ & $\mathbf{4}$ \\
\hline \multirow{3}{*}{ Circularity } & Recyclable Mass (kg) & 0 & 9 & 0 & 0 & 0.080 \\
\cline { 2 - 7 } & Reusable Mass (kg) & 0 & 0 & 0 & 300 & 0.080 \\
\cline { 2 - 7 } & Biodegradable Mass (kg) & 0 & 0 & 0.012 & 0 & 0 \\
\hline \multirow{3}{*}{ Sustainability } & Energy Consumption (MJ) & 0.885 & 2.06 & 1.024 & 38.88 & 8.58 \\
\cline { 2 - 7 } & Carbon Footprint (MJ) & 0.028 & 0.828 & 0.589 & 6.798 & 0.54 \\
\cline { 2 - 7 } & Product Cost (USD) & 0.25 & 0.1 & 0.25 & 10 & 20 \\
\hline
\end{tabular}

\subsection{Sustainability comparison between original and new design}

Graphical comparison of values shown in Table 5 is represented in Figures 3 (a) and (b) estimating a projection of one-year usage, assuming that cups are used once per day. Figure 3(a) shows values for circularity indicators. It is evident from the graph that better results are obtained from the use of porcelain and stainless steel, due to the meaningful degree of reusability involved. In the case of stainless steel, it is evident that apart from allowing the reusability of the product it also allows recycling processes. It should be clarified that in the case of circularity indicators the higher the values are, more circular the product will be. 


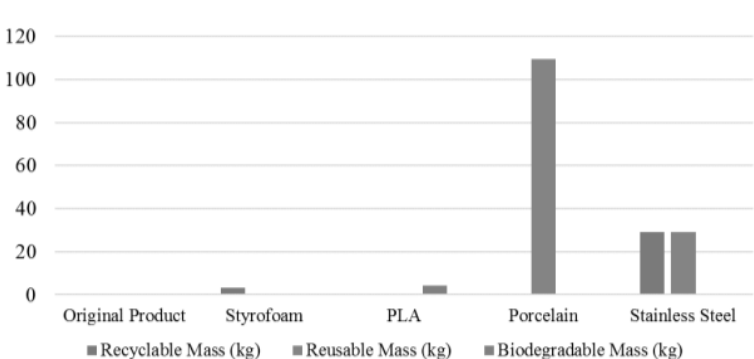

(a)

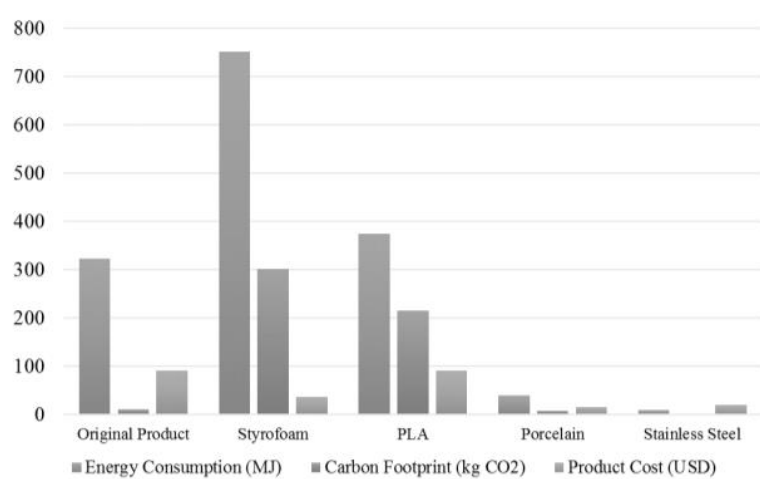

(b)

Figure 3. Circularity Indicators for one-year projection

On the other hand, Figure 3(b) shows that concerning environmental and economic sustainability, porcelain and stainless-steel alternatives material offer the least impact in a one-year projection. This is because, for a single cup, these alternatives provide higher values than Styrofoam and PLA, but given that they are reused, the environmental impact associated with obtaining the material and manufacture the product is measured once. As well as the original cup, Styrofoam and PLA alternatives generate daily waste since the product is not reusable. The same situation occurs with the acquisition cost of each alternative, although alternatives of ceramic and stainless-steel cups denote much higher values, it is only necessary to buy one and reuse it daily. To summarise, the best alternative corresponds to the use of stainless steel according to the analysis of circularity and sustainability, since it allows the reuse and recycling of the manufacturing material. The use of porcelain (ceramic mug) also offers low values in sustainability impacts. However, the recycling process is still quite complex. Although other impact factors might be considered, this approach is limited to a simple model for students with no previous experience in starting to understand the impact of the material selection during the design process in the sustainability of the product.

\section{CONCLUSIONS}

The pedagogical approach and the case study presented in this work can be introduced as a module in a design course at an introductory level. As a remarkable contribution of the proposed method, it is important to highlight that the generation of manufacturing material alternatives entails a trade-off between cost-effectiveness and long-term sustainability and provides a framework for engineering students about the non-perceptible impacts generated even in very simple situations. This approach has some limitations due to the nature of sustainability that provides future research opportunities.

\section{REFERENCES}

[1] J. Mesa, I. Esparragoza and H. Maury, "Sustainability in Engineering Education: A Literature Review of Case Study and Projects," in 15th LACCEI International Multi-Conference for Engineering Education, and Technology, Boca Ratón, USA, 2017.

[2] K. Ramani, D. Ramanujan, W. Bernstein, F. Zhao, J. Sutherland, C. Handwerker, J. Choi, H. Kim and D. Thurston, "Integrated Sustainable Life Cycle Design: A Review," Journal of Mechanical Design, vol. 136, pp. 091004-1 to 091004-15, 2010.

[3] N. M. P. Bocken, I. de Pauw, C. Bakker and B. van der Grinten, "Product design and business model strategies for a circular economy," Journal of Industrial and Production Engineering, vol. 33, no. 5, pp. 308-320, 2016.

[4] Ellen McArthur Foundation, Circularity Indicators. An Approach to Measuring Circularity. Methodology, 2010.

[5] H. Ziada, "Disposable Coffee Cup Waste Reduction Study," 15 December 2009. [Online]. Available: https://www.mcmaster.ca/sustainability/documents/DisposableCofeeCup.pdf. [Accessed 17 January 2018].

[6] Amec, "Toronto Website," 2009. [Online]. Available: http://www.toronto.ca/garbage/packaging_reduction/pdf/amec_cupsendmarket_assessment_rpt.pdf. [Accessed 17 January 2018]. 